\title{
The Distribution of the Patrol Drone
}

\author{
Junbo Lu \\ School of North China Electric Power University, Baoding 071000, China. \\ lu_ncepu@126.com
}

\begin{abstract}
In recent time, a plenty of "jaywalkers" cause threat to some law-abiding drivers in the city, and the government decides to take some measures to deal with the phenomenon. At present, they want to carry out different surveillance plans for the entire district using a certain number of drones. In order to ensure that all the geographic parts are observed in the continuously 15 minutes, I apply a goal planning model, which is based on the graph theory. According to the model, the result is calculated that 13 drones are needed and the flight route for each drone is decided. Under this condition, each drone could fly safely and accomplish the patrol task. After solving all the problems, we do a sensitivity analysis with the change of the speed. The stable drone numbers and slightly changed paths showed the robustness of our models. With more precise data and further experiment, the model can be used to provide more suitable scheme.
\end{abstract}

Keywords: jaywalker; security; graph theory; goal planning; Dijkstra algorithm; drone monitoring.

\section{Introduction}

Now, the society develops rapidly especially in economics and politics but the self-quality of many people is still worrying. In the future, the "jaywalking problem" is more severe than before in modern cities and the danger index of law-abiding drivers rises for a massive emergence of jaywalkers. In order to solve such problem, the government decides to implement a surveillance plan to the entire district of the city. To make good use of technology and effectively reduce the unnecessary waste of human resource, the government just plans to use some drones to conduct the surveillance. In fact, the current drones are robust, without recharging or refueling in 5 hours. Under this condition, the drones can follow any patrol strategy programmed by a sophisticated computerized controller without people.

\section{Some Assumptions}

1. The surveillance coverage of the drones are considered as a stable square area. In other words, when the drones carry out the surveillance plan, its scan range is distributed uniformly centered around itself. Also, the patrol coverage for every drone is limited and regarded as the same.

2. All the drones proceed at a constant speed. During the patrol, the drones will keep the same rate even encountering a turn. The average speed of the drone is 8 meters per second and I assume that it keeps forward at that rate all the time. The situation of the speed changing drones is not considered.

3. All the drones fly at the same height. To ensure that each drone has the same patrol area, we must control the same height.

4. The patrol area of each drone is regarded as a circle. The patrol area is stable and it can go ahead in a 360 degrees' view. 


\section{Symbol Explanation}

\begin{tabular}{c|c}
\hline$m$ & The number of drones accomplishing the surveillance plan. \\
\hline$x$ & The route numbers. \\
\hline$b(m)$ & The number of dots on the route $\mathrm{x}$. \\
\hline$b_{x}$ & The set of labels for all the dots on the route $\mathrm{x}$. \\
\hline$a(x)_{i}$ & The dot which is on the route $\mathrm{x}$ with a label $\mathrm{i}$. \\
\hline$i$ & The label of a dot on a route. \\
\hline
\end{tabular}

\section{Basic Model Application}

In order to facilitate the calculation, the city map is divided into grids in terms of the surveillance coverage of drones. Every grid area is exactly the surveillance coverage. And the map is divided according to a certain given scale. Taking into account the incompleteness of the city edge part, the grid is regarded as a complete grid if more than half of the area is in the district of the city. If not, it is not taken into consideration. then all the "complete grid" is labeled, starting from NO.1. In Figure 1 , it clearly shows that the city is divided into 50 grids.

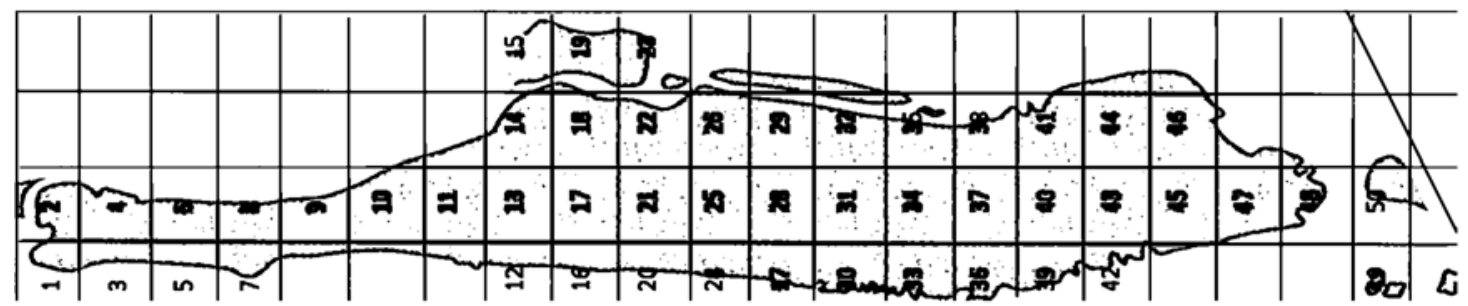

Figure 1. The map is rotated 90 degrees counterclockwise and the city part is divided into 50 grids labeled with numbers.

As the speed of the drones is all considered 8 meters per second, it will take 2 minutes for one drone to fly from one grid to another grid. I prescribe that a drone choose to fly to the surrounding 8 grids only. Here the Dijkstra algorithm is applied to find the optimal quantity of the drones. A construct empowerment graph $\mathrm{G}=(\mathrm{V}, \mathrm{E}, \mathrm{W})$ is created. For each point $\mathrm{v}$ in the map, it replaces the grid in the same position and $\mathrm{V}$ is the set of all the points. Namely:

$\mathrm{V}(\mathrm{G})=\left\{V_{1}, V_{2}, V_{3} \ldots V_{n}\right\}$ corresponds to each grid label. $\mathrm{E}(\mathrm{G})$ is the set of sides. $\mathrm{W}(\mathrm{G})$ is an adjacency matrix of $50 \times 50$. As for $\mathrm{W}(\mathrm{G})=\left(w_{i j}\right)_{m \times n}$ is defined in the following:

$$
w_{i j}\left\{\begin{array}{llll}
2 \min & v_{i} & v_{j} & \text { neighbored } \\
0 & v_{i} & v_{j} & \text { not neighbored }
\end{array}\right.
$$

In order to meet the requirement that all the sites must be observed at least once in a continuous 15 minutes, I plan that every drone patrol at a stable route. So I transform the problem into one way patrol time less than 7.5 minutes. In addition, the whole district must be monitored. That is to say, the union of all the labels should contain all the numbers. It can be showed in the following formula:

$$
\text { s.t. }\left\{\begin{array}{l}
\sum_{i=2}^{n} w_{\tilde{a}(x)_{i-1} \tilde{a}(x)_{i}} \leq 7.5 \mathrm{~min} \\
b(1) \cup b(2) \cup \cdots \cup b(m)=\{1,2,3, \cdots, 50\}
\end{array}\right.
$$


According to the time and space bound, the patrol route distribution and the corresponding quantity of the drones can be calculated. The flight paths are listed in Table 1:

Table 1. The Flight Route Distribution

\begin{tabular}{|c|c|c|c|}
\hline The number of the drones & Flight Route & The number of the drones & Flight Route \\
\hline 1 & $1->2->4->3$ & 8 & $29->32->31->30$ \\
2 & $5->6->8->7$ & 9 & $33->34->35->38$ \\
3 & $9->10->11->13$ & 10 & $36->37->40->39$ \\
4 & $12->16->17->18$ & 11 & $41->44->43->42$ \\
5 & $14->15->19->23$ & 12 & $45->46->47->48$ \\
6 & $20->21->22->26$ & 13 & $49->50->48->47$ \\
7 & $24->25->28->27$ & & \\
\hline
\end{tabular}

\section{Sensitivity Analysis}

In order to calculate the number of the drones under the condition that all the sites must be observed in a continuous 15 minutes, it is assumed that the drone goes ahead at 8 meters per second. However, we lack the precise data of the drones, therefore, we must carefully examine the effects for the final results due to the change of the speed.

First, I give a $10 \%$ interruption to the speed of the drones and compare the results with the previously recorded results. We make 2 tables to display the actual variation. Table 2 is the results that caused by a $10 \%$ rise of the speed.

Table 2. The Flight Route Distribution

\begin{tabular}{|c|c|c|c|}
\hline The number of the drones & Flight Route & The number of the drones & Flight Route \\
\hline 1 & $1->2->4->3$ & 8 & $29->32->31->30$ \\
2 & $5->6->8->7$ & 9 & $33->34->35->38$ \\
3 & $9->10->11->13$ & 10 & $36->37->40->39$ \\
4 & $12->16->17->18$ & 11 & $41->44->43->42$ \\
5 & $14->15->19->23$ & 12 & $45->46->47->48$ \\
6 & $20->21->22->26$ & 13 & $49->50->48->47$ \\
7 & $24->25->28->27$ & & \\
\hline
\end{tabular}

Compared with the table in problem 1, both the number of the drones and the flight route are basically the same, proving the model is obviously right and robust under reasonable conditions.

\section{Reference}

[1]. Ye Qixiao. 1994. Engineering Mathematics Special of Mathematical Modelling Education and Internati onal Mathematical Contest in Modeling. Journal of Engineering Mathematics.

[2]. Li Daqian. 1998. China Undergraduate Mathematical Contest in Modeling. Beijing: Higher Education Press.

[3]. Valiant, Leslie, (2013) Probably Approximately Correct: Nature's Algorithms for Learning and Prospering in a Complex World New York: Basic Books. ISBN 978-0465032716. 